\title{
Posibilidades y limitaciones en la formación académica de los
}

\section{comunicadores}

\author{
Lic. Alejandra Ledezma Lara *
}

El mundo universitario para los alumnos de la licenciatura en Ciencias de la Comunicación suele tornarse complejo. Desconocen a qué se dedicarán de manera profesional: ¿relaciones públicas?, ¿periodismo?, ¿marketing? Es una incógnita. La hibridación está presente y el momento crucial se acerca, pronto terminarán ocho semestres y se encontrarán en un ambiente laboral que en numerosas ocasiones se torna hostil.

Debo decir que la profesionalización de un alumno de comunicación depende en mucho de él o ella; un joven que carezca de la vocación para comunicar e investigar de manera responsable y veraz, poco tendrá que compartir a una sociedad necesitada de mensajes que la hagan despertar del letargo, de la manipulación y del maniqueísmo, elementos que integran la dieta diaria de millones de mexicanos.

Hace unos días la Universidad La Salle Pachuca, a través de la Escuela de Ciencias Humanas, organizó el Primer Coloquio de Comunicación; espacio propicio para la discusión de temas concernientes al desarrollo académico y profesional de los egresados de las diversas instituciones educativas que ofertan la carrera de comunicación en la ciudad de Pachuca. Así, consideré propicio realizar un brevísimo recorrido referente a la conformación de esta carrera en nuestro país; para ello seleccioné al Doctor Raúl Fuentes Navarro ${ }^{1}$ y su obra La emergencia de un campo educativo.

El objetivo de exponer los diferentes modelos de "creación" de la carrera de comunicación en México, es evidenciar que el problema del diseño curricular de esta carrera no es privativo de ninguna universidad; muchos han sido los factores que le han dado un rasgo sui generis a nuestra carrera.
El Dr. Fuentes Navarro nos recuerda que la comunicación es fundada académicamente por jesuitas en la lbero y su proyección les debe mucho. Al llegar a los setenta esa energía se había desgastado y el nuevo impulso provino de los años setenta. A México llegaron sudamericanos izquierdistas y es también la izquierda mexicana la que toma a las ciencias sociales de las universidades públicas, incluyendo obviamente a la UNAM. La combinación del componente religioso y la militancia revolucionaria, dice Fuentes Navarro, carga a la comunicación de connotaciones utópicas de reivindicación social. Es precisamente en este escenario donde han predominado tres modelos fundacionales para la formación de profesionistas en comunicación. Estos paradigmas articulan de manera diferente el currículo. Cada uno de ellos ha revestido de forma distinta a la comunicación como disciplina académica, pero ninguno de ellos ha podido legitimarse académica o profesionalmente.

El primer y más antiguo modelo, afirma el Dr.Navarro, es la formación de periodistas y es el más arraigado en las escuelas, aun en aquellas que fueron instituidas ya como escuelas de "comunicación". Tres de los rasgos constitutivos de este modelo son:

a) La prioridad de la habilidad técnico-profesional

b) El relativo ajuste a las demandas del mercado laboral

c) El propósito de la incidencia político-social a través de la opinión pública.

\footnotetext{
* Lic. Periodismo y Comunicación Colectiva por la UNAM. Maestrante en Enseñanza Superior por La Salle Pachuca. Actualmente docente de la Universidad La Salle Pachuca. alejandraledzma@yahoo.com.mx
} 


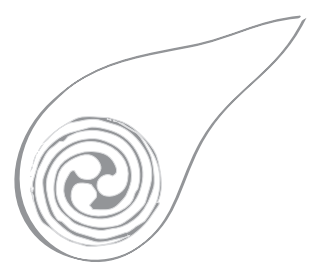

profesional aceleró sus pasos y se volvió compleja; de esta forma la vida académica se vio imposibilitada para cubrir las necesidades que demandaba el ámbito laboral. Si realizáramos una línea del tiempo; recordaremos que la profesión de la comunicación no surgió con la primeras escuelas de "comunicación"; el periodismo en nuestro país ya se ejercía desde la Independencia; así, la profesión comunicativa ya se practicaba antes de que se crearan las escuelas dedicadas a su enseñanza y se diera el estudio científico de la disciplina. Fue el rápido cambio tecnológico y el crecimiento de los medios masivos lo que desencadenó la "moda" de los estudios de comunicación; la indefinición en los perfiles de ingreso y egreso también hizo patente la indefinición del campo laboral. Sosa García menciona que en 1981 El Consejo Nacional para la Enseñanza e Investigación en Ciencias de la Comunicación, CONEICC, realizó en México un diagnóstico entre 24 escuelas de comunicación, a partir del cual identificó 3 tipos de currículas:

1) Los de corte marcadamente profesional o práctico - COMUNICANTE

2) Los que hacen énfasis en los aspectos teóri$\mathrm{Co}$, metodológicos y de investigación - COMUNICÓLOGO

3) Los que procuran equilibrar los aspectos teóricos y los prácticos - COMUNICADOR

De acuerdo a este diagnóstico, la mitad se ubicó en la primera categoría, que se caracteriza por el adiestramiento técnico encaminado a su mercado "natural" de trabajo: los medios masivos. El resto propone la formación de estudiosos de la comunicación que buscan articular teoría y práctica, pero sin definir el tipo de profesional que forman.

Uno de los problemas más arraigados es la idea de que la comunicación sólo comprende la prensa, radio y t.v., es decir, los medios masivos que desde su aparición se presentaron como el campo auténtico y natural de los egresados de esta carrera.
Ahora bien, se ha mencionado en reiteradas ocasiones la palabra comunicólogo; ahondemos en ello: desde el punto de vista de Eulalio Ferrer, existe diferencia entre comunicación y comunicología; por la primera se entiende aquel proceso activo de significación de intercambio de mensajes por el que los hombres se identifican, se influyen y se orientan hacia un fin social determinado. Por la segunda -ciencia de la comunicación en su literalidad- entendemos el conocimiento metódico de los mecanismos funcionales que determinan el modo, la orientación y la estructura fundamental de los sistemas de comunicación, en función de su transformación y de su adecuación permanente al destinatario final de ésta.

Se establece esta diferencia porque, todavía en la actualidad, existe confusión entre:

Comunicador

Comunicólogo

Licenciado en Comunicación

Sin duda alguna, el nombre más llamativo es "comunicólogo", entonces la pregunta surge ¿por qué no existe una licenciatura en comunicología?

Para el Dr. Raúl Fuentes Navarro, algunas de las dificultades que han impedido avances en la definición del currículo en comunicación se deben a:

La pretensión de trabajar únicamente con el aspecto estático y manifiesto del currículo: asignaturas y su seriación.

Desequilibrio entre las diversas asignaturas. Insuficiencia de recursos humanos, pedagógicos y materiales.

Falta de integración entre teoría y práctica.

Falta de adecuación entre formación académica y demanda del mercado profesional. 
Carencia de investigación respecto a la realidad comunicativa circundante

Con base en lo anterior, surge la imperiosa necesidad de adecuar los planes y programas de estudio de las licenciaturas en comunicación a las nuevas realidades planteadas por la práctica en comunicación que se desarrolla mucho más de prisa que los conceptos teóricos que las deben explicar; sobre todo en lo referente a los avances tecnológicos. Javier Esteinou ${ }^{2}$, argumentó que las necesidades sociales originarias de la formación universitaria de comunicadores son primeramente, en consecuencia, las que los medios hegemónicos imponen y que en síntesis consisten en preparar elementos capaces de contribuir al reforzamiento de las funciones publicitarias y la reafirmación del consenso social en torno a un modelo de desarrollo a la medida de las minorías dominantes y del capital trasnacional. Con este punto de vista, manifiesta Raúl Fuentes Navarro, las universidades han asumido posturas curriculares que pueden resumirse así:

a) Asumen y justifican la atención a estas necesidades del sistema, la mayor parte de las veces con base en una concepción reduccionista al campo de trabajo (mass-nedia, agencias de publicjdad y mercadotecnia).

b) Asumen implícitamente la misma postura, pero declaran una orientación transformadora del sistema con base en una formación humanista que incide sobre el contenido y la forma de los mensajes.

c) Ignoran toda implicación ideologicopolítica y consideran a la carrera como un programa neutral de adiestramiento tecnicocultural, cuya dimensión ética concierne sólo al individuo que se capacita.

d) Amplían el campo de acción profesional hacia otros ámbitos no masivos relacionados con las mismas funciones economicopolíticas, como el de la comunicación interna y externa de las organizaciones públicas y privadas.
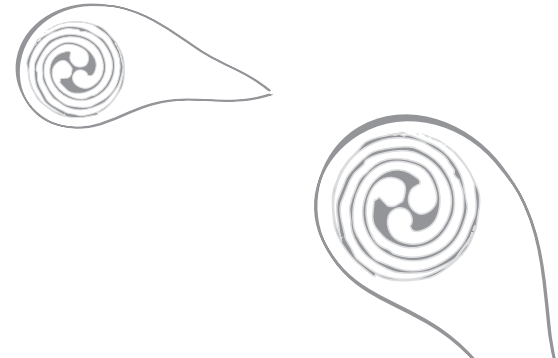

d) Rechazan la validez y legitimidad de tales necesidades y declaran como orientación el análisis crítico y una elaboración teoricopráctica que opongan alternativas a las prácticas imperantes de la sociedad.

e) Diversifican el campo de competencia de la profesión.

Lamentablemente el alumno de comunicación recibe contenidos, pero no tos integra como conocimientos; la falta de contacto/con el objeto concreto imposibilita la crítica en cuanto al grado de correspondencia eon los procesos objetivos. En un deber ser, dice el Dr. Navarro, el estudiante tendría que leer realidades para generar estrategias haciendo un uso adecuado de la tecnología en información.

Por otro lado, comparto lo establecido por Jesús-Martín Barbero ${ }^{3}$ al sugerir que un currículum-universitario en comunicación no debería estar sujeto completamente a la evolución del mercado de trabajo, ya que esto supondría que las instituciones de educación superior, sólo son instancias reproductoras del sistema social, dejando de lado su función principal: renovar críticamente la cultura y transformar las estructuras sociales.

\footnotetext{
${ }^{1}$ Documentación en Ciencias de la Comunicación, Instituto Tecnológico y de Estudios Superiores de Occidente, ITESO, Departamento de Estudios Socioculturales, Dr. Raúl Fuentes Navarro

2 Investigador Titular del Departamento de Educación y Comunicación de la Universidad Autónoma Metropolitana, Unidad Xochimilco

${ }^{3}$ Nacido en Ávila, España, en 1937. Estudió Filosofía y Letras en la universidad católica de Lovaina, Bélgica, donde se doctoró en 1971, y Antropología y Semiótica en la Escuela de Altos Estudios de París. Director del Departamento de Comunicación de la Universidad del Valle en Cali (Colombia), donde permaneció entre 1975 y 1995.
} 
Fuentes de consulta.

FERRER Rodríguez, Eulalio. (1982)

Comunicación y Comunicología. Ed. Eufesa, México

NAVARRO Fuentes, Raúl (1991) Diseño

curricular para las escuelas de comunicación.

Ed. Trillas, México

NAVARRO Fuentes, Raúl (1998) La emergencia de un campo académico, ITESO Universidad de Guadalajara

Revista electrónica Razón y Palabra febreroabril 2000

Revista electrónica Razón y Palabra. agostoseptiembre 2003

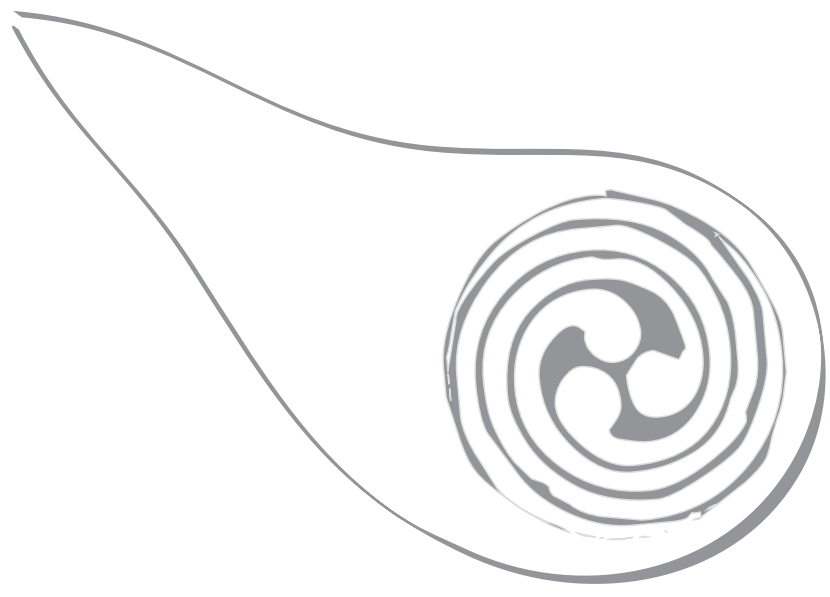

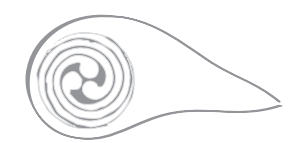
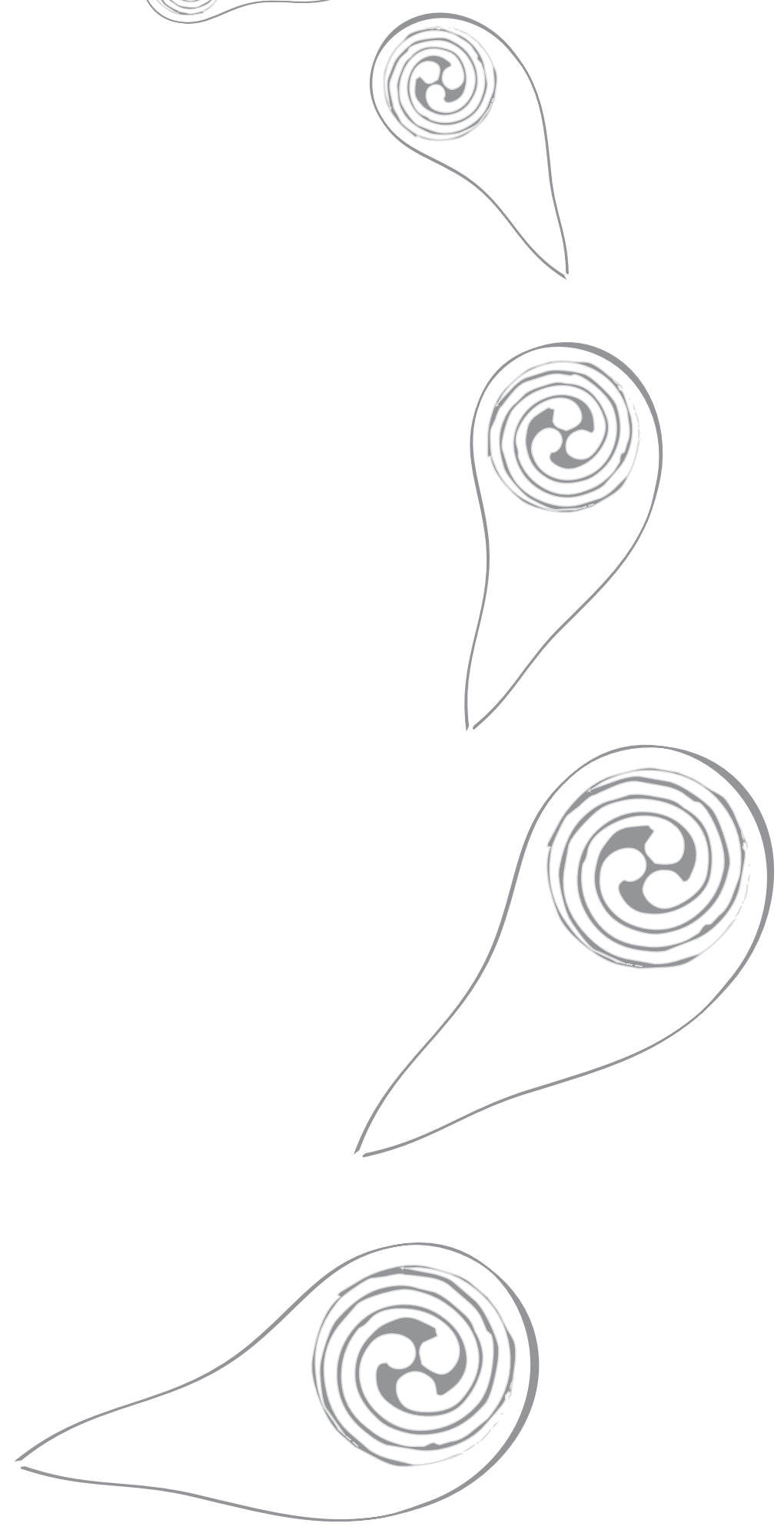